\title{
Competencias, Formación y Empleo. Análisis de Necesidades en un Programa de Master en Ingeniería
}

\author{
María J. Albert-Gómez ${ }^{(1)}$, María García-Pérez ${ }^{(1)}$ y Clara Pérez-Molina ${ }^{(2)}$ \\ (1) UNED. Departamento de Teoría de la Educación y Pedagogía Social. Calle Juan del Rosal, 14, 28040 \\ Madrid - España (e-mail: mjalbert@edu.uned.es; mgarcia@edu.uned.es) \\ (2) UNED. Departamento Ingeniería eléctrica, electrónica y de control. Calle Juan del Rosal, 14, 28040 \\ Madrid-España (e-mail: clarapm@ieec.uned.es)
}

Recibido Jun. 23, 2016; Aceptado Sep. 1, 2016; Versión final Oct. 5, 2016, Publicado Abr. 2017

\begin{abstract}
Resumen
El objetivo de este trabajo es complementar la formación encaminada al empleo, de los graduados en Ingeniería de Sistemas y se analizaron las valoraciones sobre competencias genéricas. Se utilizó una metodología empírica descriptiva basada en la observación, en el análisis documental y cuestionarios, utilizando datos cuantitativos y cualitativos. Se realizó un análisis de necesidades, se identificaron las competencias específicas y se montaron cursos específicos para desarrollarlas dentro de un programa de Master. Los resultados evidencian la necesidad de mejorar el currículo de los ingenieros en las competencias: capacidades técnicas y específicas, dominio de su área/disciplina; pensamiento analítico; capacidad para utilizar herramientas informáticas; capacidad para escribir y hablar en idiomas extranjeros; capacidades técnicas y de orientación a resultados, concentración y abstracción, orientación de calidad y habilidades internacionales. En conclusión se puso de manifiesto la necesidad de desarrollar una serie de competencias clave a través de la formación de postgrado.
\end{abstract}

Palabras clave: competencias; empleabilidad; análisis de necesidades; desarrollo curricular; formación.

\section{Skills, Training and Employment. Need Analysis in a Master Program of Engineering}

\begin{abstract}
The aim of this paper is to complement the training of the graduates in system and automatic engineering leading to employment. A descriptive methodology based on empirical observation, document analysis and questionnaires, using quantitative and qualitative data were used. A need analysis was done on them, the specific skills were identified and specific courses were set up to develop them in a Master program. The results show the need of improving the curriculum for training and employment of engineers in the following skills: technical and specific skills, command of their area or discipline; analytic thought; ability to use computer tools; ability to write and speak in foreign languages; technical abilities and result orientation (concentration and abstraction, quality and orientation skills) and international capabilities. In conclusion the need to develop a number of key skills through postgraduate training of engineering graduates was highlighted.
\end{abstract}

Keywords: competence; employability; need analysis; curriculum development; training 


\section{INTRODUCCIÓN}

Las universidades, las empresas y, en definitiva, el mundo laboral, se enfrentan a nuevas necesidades derivadas de la sociedad del conocimiento donde están insertos. Una sociedad basada en aprender a aprender, adquirir nuevas competencias digitales, para poder hacer frente a los nuevos acontecimientos en un entorno en constante cambio. La globalización de los mercados, la internalización profesional, la creciente rapidez con la que se producen los cambios tecnológicos exigen nuevos profesionales formados no sólo en el conocimiento teórico sino también en el desarrollo de habilidades y competencias, esto nos lleva a pensar en la formación de los graduados en una realidad concreta donde adquiere gran protagonismo el concepto de empleabilidad (Agudo et al, 2013).

En este contexto, las áreas de trabajo, las competencias necesarias de las personas, en general, y en este caso específico de los ingenieros de la rama de ingeniería de sistemas y automática, evolucionan rápidamente obligándoles a los empleados a un proceso continuo de adaptación para mantenerse día a día en el mercado laboral cambiante. En este ambiente transformador, las Universidades y las Instituciones educativas tienen que desarrollar y promover los cambios descubriendo en ellos el verdadero sentido y equilibrio entre lo que se aprende y la practicidad de lo aprendido. En este sentido, las universidades se enfrentan a nuevos retos, ya que deben preparar a sus estudiantes ofreciéndoles una base formativa y competencial que cubra las nuevas demandas del mundo laboral de hoy en día. Para ello, las universidades y las empresas deben ser capaces de adquirir una comprensión completa de las necesidades del mercado actual en áreas emergentes y ofrecer justo a tiempo, y justo lo suficiente, un aprendizaje flexible, adaptado exclusivamente a las necesidades de los estudiantes y su futuro puesto de trabajo (Caldas, Lacalle y Carrión, 2012), constituyéndose así una formación activa, solida, bien estructurada, consensuada y debidamente planificada para contribuir a un aumento de la productividad y a un crecimiento económico.

En base a lo señalado, y teniendo en cuenta que en ocasiones el sistema educativo es, en general, relativamente estable y en ocasiones impermeable, la formación de postgrado se presenta como factor clave en la sociedad basada en el conocimiento, donde hay una necesidad de formación continua como un proceso de reciclaje continuo donde los trabajadores deben constantemente ser capaces de aprender asegurando un conocimiento práctico sólido (Carozo, 2013; Martin et al, 2013). Las empresas cada vez más evalúan a sus empleados a través de competencias, lo que según Bustamante et al (2015) el nivel de competencias constituye un diferenciador crítico del rendimiento. En este sentido, las universidades tienen puesta su mirada en la inserción laboral de los egresados considerando que un universitario es más empleable cuanto mayor es su capacidad para adaptar sus competencias a las demandas del mercado laboral.

El concepto de competencias es amplio y variado, así podemos destacar que es atribuido a unas características subyacentes de un individuo que están causalmente relacionadas con el logro de un rendimiento en el trabajo efectivo o superior, sin embargo, para otros como Blanceroet al. (1996) o Pimienta (2011) las competencias deben de ser entendidas como los conocimientos, destrezas, habilidades y otras características requeridas para el desempeño del trabajo efectivo. Por su parte, Schmal (2015) entiende la competencia como la movilización, la puesta en acción de un conjunto de capacidades cognitivas, procedimentales y actitudinales que se deben integrar en contextos específicos. A pesar de las diferentes definiciones encontradas y la evolución del término, todos ellos comparten que la competencia se refiere a un conjunto de atributos, que incluyen los conocimientos, destrezas, habilidades y rasgos, necesarios y relacionados con la finalización del desempeño laboral, pero teniendo presente que las competencias tienen características específicas de la región (Sgobbi y Suleman, 2013; Peng, Shulin y Gu, 2014).

El objetivo del presente estudio es determinar las competencias genéricas y específicas a desarrollar y las necesidades formativas de los ingenieros de la rama de ingeniería de sistemas y automática para montar un Master en esa rama y fomentar las potencialidades para el empleo. Se utilizará una metodología empírica descriptiva basada en la observación, análisis documental y cuestionarios con un diseño cuasi experimental.

\section{EL PROYECTO PAC}

El proyecto Performance-centred Adaptative Curriculum for Employment Needs (en lo sucesivo PAC) ha sido diseñado para contribuir a los cambios y nuevas demandas de la sociedad relacionados con los procesos de aprendizaje a través de un plan de formación basado en competencias según se indica en el objetivo anteriormente marcado. Este planteamiento se basó en la fundamentación y en los antecedentes del propio proyecto PAC por un lado y, por otro lado, en la fundamentación y antecedentes de distintos estudios y autores que manifiestan, entre otras, la importancia de una conexión entre el desarrollo de competencias y el mercado laboral en este caso de los ingenieros de la rama de Ingeniería de Sistemas y Automática. 
Martin et al. (2013), señala que se han producido en la última época transformaciones en el mundo laboral que no han tenido un reflejo curricular en las instituciones educativas, y por esta razón, Estepa y Estepa $(2011,214)$ afirman que se ha producido una "desconexión con las competencias demandadas a los ingenieros en la actualidad". Rugarcia et al. (2000) señalan algunos elementos que han influido en un claro cambio de contexto laboral en los últimos años: inundación de información y accesibilidad pública a través de Internet, gran desarrollo de tecnologías multidisciplinares, mercados globalizados, estructuras corporativas más participativas y cambios muy rápidos. En este sentido, Rué (2009) asegura que es necesario que se incluyan una serie de competencias profesionales dentro del currículum académico, que según este autor deben centrarse en torno al: aprendizaje autónomo, la creatividad, el pensamiento crítico y resolución de problemas, habilidades de comunicación social, trabajo en grupo.

Según la encuesta de Farrell et al. (2005) el 80,7\% de los graduados de ingeniería en los EE.UU. eran competitivos a nivel mundial, mientras que muchos de los países investigados revelaban que tan solo el $10 \%$ estaban capacitados y eran competitivos en el mundo globalizado. Esta investigación refuerza la necesidad de la adaptación curricular de los ingenieros centrándose en el desarrollo de las competencias profesionales, que en palabras de Peng, Shulin y $\mathrm{Gu}(2014,1)$ se resume en "la exploración de las diferencias entre el cultivo de competencias en materia de educación y las necesidades de la industria es esencial".

Existen numerosas investigaciones que se han llevado a cabo durante los últimos tiempos en torno a este problema, principalmente en Australia (Ashman et al. 2008; Nair, Patil, y Mertova, 2009), China (Peng, Shulin y Gu, 2014), Europa (Bodmer et al., 2002) y otros estudios de carácter internacional donde se comparan diferentes países y regiones (Downey, Lucena, y Mitcham, 2007; Lucena et al, 2008; OCDE, 2011). Sin embargo, a pesar de las múltiples investigaciones los resultados en gran parte de ellas destacaron que las competencias profesionales necesarias de los ingeniería desarrolladas a través de los master en ingeniería variaron en los diferentes países, de ahí la importancia de desarrollar la investigación en una región concreta, como puede ser Europa donde se está avanzando en la implementación total del Espacio Europeo de Educación Superior (en lo sucesivo EEES).

Como señala Martin et al (2013) "una de las misiones fundamentales que tiene encomendada la universidad es la de proporcionar una formación y cualificación suficiente que garantice la empleabilidad (employability) y competitividad de sus titulados y, por tanto, que permita la inserción laboral plena de los mismos". Sin embargo, dentro del contexto europeo, muchos de los sistemas de aprendizaje propuestos por la universidades no consiguen sus objetivos (OCDE, 2011), principalmente por tres razones: por no capturar la demanda de nuevos conocimientos dejando sin actualizar los cursos y los programas correspondientes; el aprendizaje no siempre se traduce en ideas prácticas por lo que puede haber diferencias en situaciones reales de trabajo; no se produce una adaptación de la formación a grupos más amplios de edad, por lo que se deja fuera a estudiantes con empleo que provienen de las empresas. Según HeyWood (2005), las universidades conscientes de estos problemas y, con el fin de responder a la necesidad de una formación masiva, amplia y dirigida a un mayor número de estudiantes de todas las edades y situaciones, están tratando de adoptar este enfoque, impulsado por la necesidad de aprender y poner en práctica lo aprendido y proporcionar medios a través de los cuales se pueden superar los problemas detectados. Piezas importantes para superar las necesidades y los problemas detectados son las actividades conjuntas entre las empresas y la universidad a niveles regionales, nacionales e internacionales y sectorial (Peng, Shulin y $\mathrm{Gu}, 2014)$.

Para poder cumplir con las exigencias y necesidades que se destacaban, fase puso en marcha un proyecto de investigación en el que participaron tanto las empresas como las universidades a nivel europeo, con el objetivo de desarrollar y adaptar el plan de estudios de los ingenieros de la rama de sistemas y automática a nivel de Máster a las necesidades de empleo. En este proyecto participaron Empresas y Universidades. Las empresas participantes son: Adaptative Predictive Expert Control ADEX, S.L. de España;Techn. Enhanced Performance Oriented Learning Soft - TEPOLSOFTS de Bulgaria; International Software Consulting Network Gesmbh de Austria. Las universidades participantes son: Plovdiv University "Paisii Hilendarski" - Bulgaria; Universidad Nacional de Educación a Distancia - UNED - España. Departamento de Ingeniería eléctrica, electrónica y de control DIEEC; Departamento de Teoría de la Educación y Pedagogía Social.

El objetivo general es adaptar el plan de estudios de ingeniería en la rama de sistemas y automática a nivel de Máster a las necesidades de empleo. Los objetivos específicos son: Determinar las competencias genéricas y específicas; Identificar las nuevas demandas de cualificación en el ámbito de la Ingeniería de Sistemas y automática (ISA); Proporcionar a nuestros profesionales una formación teórica-práctica a través a nivel de Master que les conceda una cualificación adecuada para ser competitivos en el mercado de trabajo; Comprobar el grado de satisfacción de los estudiantes con los cursos en el Master. 


\section{METODOLOGÍA}

La muestra con la que se trabajó responde a los tres colectivos con los que se trabaja en la investigación: 15 empleadores, 15 recién graduados y 17 profesores. Se utilizó un diseño cuasi experimental y con un muestreo no probabilístico intencional, ya que la elección de la muestra no depende de la probabilidad, sino según se estime que son representativos o típicos de la población, así se ha trabajado con una población finita. Se utilizó una metodología empírica descriptiva basada en la observación, en el análisis documental y cuestionarios, utilizando procedimientos concurrentes en los cuales convergen datos cuantitativos y cualitativos, utilizando las fortalezas de cada uno de ellos para responder a distintas cuestiones de investigación de modo que la comprensión e interpretación de la realidad se enriquece gracias a la integración de ambos tipos de datos. Para el análisis de los datos cuantitativos se utilizó el programa estadístico SPSS versión 21.

El primer paso de la investigación fue la detección de las competencias y necesidades formativas es imprescindible para que un plan de formación sea realista y se ajuste a los intereses de las empresas y de los estudiantes, ha de ser un proceso sistemático que abarque a todos los implicados. En un primer momento se realizó de una revisión sistemática de la literatura existente en este campo lo cual se manejaron dos de las bases de datos más importantes de publicación científica a nivel internacional, como son ISI Web of Science (WoS) y SCOPUS, analizando artículos comprendidos entre los años 2005 y 2015, así como un estudio exploratorio de informes relevantes en el campo de la ingeniería que tratan sobre el ámbito de las competencias básicas y transversales necesarias para el empleo y desarrollo laboral de los ingenieros en Australia, India, Singapur, Europa, el Reino Unido y EE.UU. Los documentos analizados fueron los de ABET (2009), Tuning (2005), EAAB (2005), IES (2004), ECUK (2003), y NBA (2000). Estos documentos servirían de base para establecer las competencias necesarias a desarrollar y que deben estar fundamentados en las exigencias del EEES donde ya se encuentran analizadas y valoradas las competencias por los respectivos organismos europeos. Al analizar los documentos de la Unión Europea y la información sobre competencias que ofrecen los documentos señalados se observaron grandes coincidencias respecto a las competencias que destacaban como necesarias para la formación universitaria y el empleo.

El siguiente paso fue analizar las valoraciones que daban a las competencias recogidas por los profesores, graduados y empleadores relacionadas con la consecución de un empleo y las necesidades formativas en las competencias indicadas. Para este fin se elaboró un cuestionario que contenía dos partes, en una primera parte los sujetos tenían que valorar las competencias indicadas en la tabla con una valoración .En la segunda parte del cuestionarios los sujetos tenían que valorar, a su juicio, el grado de consecución de las competencias indicadas, realizando así un análisis de necesidades según profesores, empleadores y graduados. Una vez realizadas las medias, se comprobará si existían o no diferencias estadísticamente significativas entre las medias y así comprobar si la selección de nuestras competencias era la correcta. Puesto que contábamos con grupos equilibrados y varianzas similares, se procederá a realizar el análisis de la diferencia honestamente significativa de Tukey (HSD de Tukey) llevando a cabo una comparación múltiple entre los diferentes grupos de sujetos. Los resultados reflejaron que las diferencias entre los grupo expuestos no eran significativas ya que todos los resultados eran inferiores a 0,05 .

En un tercer momento, y una vez detectadas las competencias mejor valoradas y las necesidades formativas, se pidió a los profesores de las Universidades participantes en el proyecto PAC que determinaron las competencias específicas y los contenidos de los cursos que, a su juicio, complementase la formación dada en el Grado de Ingeniería en la rama de la Ingeniería de Sistemas y Automática para crear un Master como complemento formativo. A partir de aquí se realizará como prueba estadística un análisis de medias de cada uno de los colectivos para encontrar/comprobar si las diferencias son o no significativas. Y en un cuarto paso se realizó un estudio piloto poniendo en marcha algunos de los cursos que formarían parte del Master midiendo el grado de satisfacción de los alumnos, para ello se utilizaron distintos indicadores: Expectativas y motivación; Utilidad; Contenido y método utilizado en de los cursos formativos; y Herramientas utilizadas en los cursos. Las expectativas y la motivación se midieron, en la fase piloto antes y después de que los estudiantes realizasen los cursos formativos. Toda esta información aporta datos para planificar la formación y mejorar el aprendizaje de los alumnos a través de las nuevas tecnologías (Jiménez et al, 2013).

Para medir la utilidad nos centramos en la idea del aprendizaje significativo. Este tipo de aprendizaje se caracteriza por el hecho de que el alumno vaya gestionando y edificando su aprendizaje como una escalera de tal forma que un escalón se basa en el otro. Para esto es muy importante que el alumno considere útil todo aquello que hace en el proceso educativo. Otras investigaciones, como la elaborada por Marchisio y otros (2011), han utilizado este mismo indicador con resultados similares. Con la medición de este indicador se buscó conocer el grado de utilidad que conceden los estudiantes a la formación recibida, y en caso de obtener resultados negativos, se actuará para cambiar y mejorar de esta forma la formación y el aprendizaje. 
Con la medición de los Contenidos y el método utilizado se comprobó cual era la opinión de los estudiantes en cuanto a los contenidos que estudiaban y el método que se había utilizado. Las Herramientas fueron otro indicador a controlar, así otras investigaciones llegan a resultados similares y centrándose en gran medida en la influencia de las herramientas en mejora de los resultados de aprendizaje, así, por ejemplo, el estudio elaborado por Barrios, Lencina y Marín (2014) quienes a través de la modalidad de enseñanza mixta evaluaron las mejores herramientas dentro de un software de laboratorio remoto; y otras investigaciones implementaron un laboratorio para el aprendizaje de comunicaciones digitales usando plataformas reconfigurables y/o software de instrumentación y procesamiento digital de señales (Fiad y Galarza, 2015). Se consideró importante que estos indicadores fuesen medidos en distintos momentos distintos antes de realizar los cursos, en el proceso formativo y después del proceso formativo. Y finalmente, el último paso fue la inclusión del Master dentro del Plan de estudio del Grado de Ingeniería en la rama de la Ingeniería de Sistemas y Automática (ISA).

Como materiales e instrumentos se utilizó el análisis documental que sirvió de base para la elaboración de los cuestionarios elaborados para los empleadores y recién graduados. Los cuestionarios fueron elaborados en base a partir de las competencias recogidas en el análisis documental. El objetivo del cuestionario fue detectar por parte de los empleadores y graduados, las competencias y las necesidades formativas para el desempeño de una labor eficiente y eficaz. Para este fin se elaboró un cuestionario que contenía dos partes, cada una de ellas con 24 ítems en una primera parte los sujetos tenían que valorar las competencias indicadas en la tabla con una valoración entre "1" y "4" donde "1" era el valor más bajo y "4" el más alto. En la segunda parte del cuestionario los sujetos tenían que valorar, a su juicio, el grado de consecución de las competencias indicadas, la puntuación era de "1" a "3" donde "1" era competencia conseguida, "2" no conseguida y "3" necesita mejorar, realizando así un análisis de necesidades según profesores, empleadores y graduados. En la segunda parte de la investigación se pretendía evaluar el grado de satisfacción de los alumnos con los cursos que formaban parte del Master y se utilizaron cuestionarios que abarcaba los indicadores propuestos. Los instrumentos utilizados para medir los distintos indicadores fueron: Cuestionarios para determinar las competencias genéricas y necesidades formativas que se pasó a empleadores, graduados y profesores; Cuestionarios sobre el grado de satisfacción de estudiantes sobre los cursos realizados.

Para el estudio de la validez de los cuestionarios se definió la validez de contenido como el grado en que una prueba representa de forma adecuada lo que se ha realizado (Thomas y Nelson, 2007). Para alcanzar niveles óptimos de validez de contenido se utilizó la técnica de expertos. Se solicitó a los jueces de expertos que valorasen cada uno de los ítems de forma individual y los cuestionarios de forma global. Las puntuaciones sobre cada ítem eran entre 1 y 10. Se desestimaron todos aquellos ítems que tuvieron una puntuación por debajo de 8. Para el análisis de las preguntas abierta se realizó un análisis de contenido. Una vez terminado el cuestionario se pasó a los mismos estudiantes en dos momentos distintos, test, retest con un coeficiente de fiabilidad de Alfa de Cronbach de 0.90 .

\section{RESULTADOS}

En un primer momento, los resultados obtenidos de la revisión sistemática de la literatura existente y del análisis documental arrojaron una lista de competencias que fueron la base sobre la que se construirían los cuestionarios de la investigación. Dicha lista de competencias fueron:

Tabla 1: Lista de competencias que son necesarias para el desarrollo profesional de los ingenieros

\begin{tabular}{|l|}
\hline 1.-Capacidades técnicas y específicas (Dominio de su área o disciplina) \\
\hline 2.- Capacidad de negociación \\
\hline 3.- Capacidad para redactar informes y documentos \\
\hline 4.- Pensamiento analítico \\
\hline 5.- Capacidad para sintetizar y extraer conclusiones generales \\
\hline 6.- Capacidad de gestión del tiempo \\
\hline 7.- Capacidad para utilizar herramientas informáticas \\
\hline 8.- Capacidad para escribir y hablar en idiomas extranjeros \\
\hline 9.- Capacidad para detectar nuevas oportunidades, soluciones y resolución de problemas \\
\hline 10.- Capacidad para adquirir con rapidez nuevos conocimientos de forma autónoma y creativa \\
\hline 11.- Habilidades de organización \\
\hline 12.- Iniciativa y motivación \\
\hline 13.- Capacidad de tomar decisiones \\
\hline 14.- Autocontrol y capacidades de autogestión \\
\hline
\end{tabular}


Tabla 1 (continuación)

15.- Capacidad para trabajar bajo control

16.- Capacidad de crítica y autocrítica

17.- Responsabilidad

18.- Comunicación y habilidades interpersonales (Capacidad de comunicación y habilidades sociales, capacidad de liderazgo y coordinación)

19.- Capacidades técnicas y de orientación a resultados (Concentración y abstracción, orientación de calidad)

20.- Habilidades de colaboración (Trabajo en equipo, trabajo de forma remota)

21.- Valores sociales

22.- habilidades de gestión del cambio (Flexibilidad, polivalencia y adaptación al cambio, resilencia. Aprendizaje constante y curiosidad, intraemprendimiento, creatividad e innovación)

23.- Habilidades internacionales

24.- Gestión de la imagen propia

En un segundo momento, y a partir de los datos obtenidos de la lista de competencias del análisis documental surgen una parte de los datos de los resultados de esta investigación que se muestran en la Tabla 2 y en las figuras 1 a 3 .

Tabla 2. Lista de competencias valoradas por los tres colectivos que son necesarias para el desarrollo profesional de los ingenieros

\begin{tabular}{|c|c|c|c|}
\hline Competencia & Profesores & Empleadores & Graduados \\
\hline 1.-Capacidades técnicas y específicas (Dominio de su área o disciplina) & 3,5 & 3,6 & 3,7 \\
\hline 2.- Capacidad de negociación & 2,9 & 3,3 & 2,1 \\
\hline 3.- Capacidad para redactar informes y documentos & 3,2 & 2,5 & 2,3 \\
\hline 4.- Pensamiento analítico & 3,7 & 3,6 & 3,1 \\
\hline 5.- Capacidad para sintetizar y extraer conclusiones generales & 3,5 & 2,7 & 2,4 \\
\hline 6.- Capacidad de gestión del tiempo & 3,5 & 3,3 & 2,1 \\
\hline 7.- Capacidad para utilizar herramientas informáticas & 3,4 & 3,7 & 4 \\
\hline 8.-Capacidad para escribir y hablar en idiomas extranjeros & 3 & 3,7 & 3,7 \\
\hline $\begin{array}{l}\text { 9.- Capacidad para detectar nuevas oportunidades, soluciones y resolución } \\
\text { de problemas }\end{array}$ & 3,1 & 3,2 & 2,5 \\
\hline $\begin{array}{l}\text { 10.- Capacidad para adquirir con rapidez nuevos conocimientos de forma } \\
\text { autónoma y creativa }\end{array}$ & 3,4 & 3,1 & 2,9 \\
\hline 11.- Habilidades de organización & 3,3 & 2,7 & 2 \\
\hline 12.- Iniciativa y motivación & 3,2 & 3,7 & 1,9 \\
\hline 13.- Capacidad de tomar decisiones & 3,1 & 3 & 2,5 \\
\hline 14.- Autocontrol y capacidades de autogestión & 3,4 & 2,4 & 3 \\
\hline 15.- Capacidad para trabajar bajo control & 3 & 2,8 & 2,3 \\
\hline 16.- Capacidad de crítica y autocrítica & 3,4 & 3 & 2,7 \\
\hline 17.- Responsabilidad & 3,3 & 3,5 & 3,2 \\
\hline $\begin{array}{l}\text { 18.- Comunicación y habilidades interpersonales (Capacidad de } \\
\text { comunicación y habilidades sociales, capacidad de liderazgo y coordinación) }\end{array}$ & 3,1 & 2,7 & 3,2 \\
\hline $\begin{array}{l}\text { 19.- Capacidades técnicas y de orientación a resultados (Concentración y } \\
\text { abstracción, orientación de calidad) }\end{array}$ & 3 & 3,5 & 4 \\
\hline $\begin{array}{l}\text { 20.- Habilidades de colaboración (Trabajo en equipo, trabajo de forma } \\
\text { remota) }\end{array}$ & 3,3 & 2,8 & 3,7 \\
\hline 21.- Valores sociales & 3,2 & 2,5 & 2,1 \\
\hline $\begin{array}{l}\text { 22.- habilidades de gestión del cambio (Flexibilidad, polivalencia y } \\
\text { adaptación al cambio, resilencia. Aprendizaje constante y curiosidad, } \\
\text { intraemprendimiento, creatividad e innovación) }\end{array}$ & 3,3 & 2,6 & 3,8 \\
\hline 23.- Habilidades internacionales & 2,7 & 3,7 & 3,7 \\
\hline 24.- Gestión de la imagen propia & 2,9 & 2,5 & 2 \\
\hline
\end{tabular}


Según los datos obtenidos en la anterior tabla 2 se consideraron como competencias más importantes, por las medias analizadas, aquellas que tuvieran una puntuación media entre 3,5 y 4 . Según este criterio se pudo comprobar como los tres colectivos consideran como muy importantes la competencia no1.Capacidades técnicas y específicas, Dominio de su área o disciplina. Por su parte profesores y empleadores coinciden además en la competencia no 4.- Pensamiento analítico. Además empleadores y graduados coinciden en la competencia 7.- Capacidad para utilizar herramientas informáticas; en la no 8.- Capacidad para escribir y hablar en idiomas extranjeros, en la $\mathrm{n}$ ำ19.- Capacidades técnicas y de orientación a resultados, concentración y abstracción, orientación de calidad; y en la ํㅜ 23 Habilidades internacionales.

Para poder completar el estudio se tuvieron en cuenta en segunda lugar aquellas competencias cuyas puntuaciones medias estaban entre 3 y 3,5, esas competencias fueron: Capacidad de negociación; Capacidad para redactar informes y documentos; Capacidad para detectar nuevas oportunidades y resolución de problemas; Capacidad para adquirir con rapidez nuevos conocimientos de forma autónoma y creativa; Iniciativa y motivación; Habilidades para tomar decisiones; Habilidades de autocontrol y autogestión; Capacidades de crítica y autocrítica; Responsabilidad; Habilidades de comunicación interpersonal y capacidad de liderazgo; Habilidades de colaboración trabajo en equipo y de forma remota; y Habilidades para la gestión del cambio. Con respecto a las necesidades formativas analizamos las relacionadas con las competencias mejor valoradas entre los tres colectivos. Los resultados se muestran en la siguiente Tabla 3.

Tabla 3: Resultados análisis de necesidades por competencias

\begin{tabular}{|c|c|c|c|c|c|}
\hline \multicolumn{5}{|c|}{ Competencia no 1.- Capacidades técnicas y específicas (Dominio de su área o disciplina) } & \multirow[b]{2}{*}{ Total } \\
\hline & & $\begin{array}{c}\text { Conseguida } \\
\text { (1) }\end{array}$ & No conseguida (2) & $\begin{array}{c}\text { Necesita mejorar } \\
\text { (3) }\end{array}$ & \\
\hline \multirow{3}{*}{ Sujetos } & Profesores & 9 & 5 & 3 & 17 \\
\hline & Graduados & 8 & 0 & 7 & 15 \\
\hline & Empleadores & 0 & 5 & 10 & 15 \\
\hline \multicolumn{2}{|l|}{ Total } & 17 & 10 & 20 & 47 \\
\hline \multicolumn{2}{|c|}{$\begin{array}{|ll|}\text { Porcentajes (\%) } \\
\end{array}$} & $36 \%$ & $21 \%$ & $43 \%$ & $100 \%$ \\
\hline \multicolumn{5}{|c|}{ Competencia no 4.- Pensamiento analítico. } & \multirow[t]{2}{*}{ Total } \\
\hline & & $\begin{array}{c}\text { Conseguida } \\
\text { (1) }\end{array}$ & $\begin{array}{c}\text { No conseguida } \\
(2)\end{array}$ & $\begin{array}{l}\text { Necesita mejorar } \\
\text { (3) }\end{array}$ & \\
\hline \multirow{3}{*}{ Sujetos } & Profesores & 8 & 5 & 4 & 17 \\
\hline & Graduados & 11 & 3 & 1 & 15 \\
\hline & Empleadores & 11 & 4 & 0 & 15 \\
\hline \multicolumn{2}{|l|}{ Total } & 30 & 12 & 5 & 47 \\
\hline \multicolumn{2}{|c|}{ Porcentajes (\%) } & $64 \%$ & $25,5 \%$ & $10,5 \%$ & $100 \%$ \\
\hline \multicolumn{5}{|c|}{ Competencia nº 7.- Capacidad para utilizar herramientas informáticas. } & \multirow[t]{2}{*}{ Total } \\
\hline & & $\begin{array}{c}\text { Conseguida } \\
\text { (1) }\end{array}$ & $\begin{array}{c}\text { No conseguida } \\
(2)\end{array}$ & $\begin{array}{c}\text { Necesita mejorar } \\
\text { (3) }\end{array}$ & \\
\hline \multirow{3}{*}{ Sujetos } & Profesores & 9 & 3 & 5 & 17 \\
\hline & Graduados & 8 & 1 & 6 & 15 \\
\hline & Empleadores & 0 & 1 & 14 & 15 \\
\hline \multicolumn{2}{|c|}{$\begin{array}{l}\text { Total } \\
\text { Porcentajes (\%) } \\
\end{array}$} & $\begin{array}{c}17 \\
36 \% \\
\end{array}$ & $\begin{array}{c}5 \\
11 \% \\
\end{array}$ & $\begin{array}{c}25 \\
53 \% \\
\end{array}$ & $\begin{array}{c}47 \\
100 \% \\
\end{array}$ \\
\hline \multicolumn{5}{|c|}{ Competencia no 8.- Capacidad para escribir y hablar en idiomas extranjeros. } & \multirow[b]{2}{*}{ Total } \\
\hline & & $\begin{array}{c}\text { Conseguida } \\
\text { (1) }\end{array}$ & $\begin{array}{c}\text { No conseguida } \\
(2)\end{array}$ & $\begin{array}{c}\text { Necesita mejorar } \\
\text { (3) }\end{array}$ & \\
\hline \multirow{3}{*}{ Sujetos } & Profesores & 1 & 10 & 6 & 17 \\
\hline & Graduados & 6 & 0 & 9 & 15 \\
\hline & Empleadores & 1 & 4 & 10 & 15 \\
\hline \multicolumn{2}{|c|}{ Total } & 8 & 14 & 25 & 47 \\
\hline \multicolumn{2}{|c|}{ Porcentajes (\%) } & $17 \%$ & $30 \%$ & $53 \%$ & $100 \%$ \\
\hline \multicolumn{5}{|c|}{$\begin{array}{c}\text { Competencia no 19.- Capacidades técnicas y de orientación a resultados (Concentración y abstracción, } \\
\text { orientación de calidad). }\end{array}$} & \multirow[t]{2}{*}{ Total } \\
\hline & & $\begin{array}{c}\text { Conseguida } \\
(1)\end{array}$ & $\begin{array}{c}\text { No conseguida } \\
(2)\end{array}$ & $\begin{array}{l}\text { Necesita mejorar } \\
(3)\end{array}$ & \\
\hline \multirow{3}{*}{ Sujetos } & Profesores & 9 & 2 & 6 & 17 \\
\hline & Graduados & 7 & 1 & 7 & 15 \\
\hline & Empleadores & 6 & 4 & 5 & 15 \\
\hline
\end{tabular}


Tabla 3 (continuación)

\begin{tabular}{|c|c|c|c|c|c|}
\hline & Total & 22 & 7 & 18 & 47 \\
\hline Porcent & (\%) & $47 \%$ & $15 \%$ & $38 \%$ & $100 \%$ \\
\hline \multicolumn{5}{|c|}{ Competencia $n^{\circ}$ 23.- Habilidades internacionales. } & \multirow[b]{2}{*}{ Total } \\
\hline & & $\begin{array}{c}\text { Conseguida } \\
\text { (1) }\end{array}$ & $\begin{array}{c}\text { No conseguida } \\
(2)\end{array}$ & $\begin{array}{l}\text { Necesita mejorar } \\
\text { (3) }\end{array}$ & \\
\hline \multirow{3}{*}{ Sujetos } & Profesores & 3 & 2 & 12 & 17 \\
\hline & Graduados & 6 & 2 & 7 & 15 \\
\hline & Empleadores & 3 & 0 & 12 & 15 \\
\hline \multicolumn{2}{|l|}{ Total } & 12 & 4 & 31 & 47 \\
\hline \multicolumn{2}{|c|}{ Porcentajes (\%) } & $25,5, \%$ & $8,5 \%$ & $66 \%$ & $100 \%$ \\
\hline
\end{tabular}

Observando la tabla 3 , en la competencia $n^{\circ} 1$ "Capacidades técnicas y específicas (Dominio de su área o disciplina)", se puede comprobar como el $36 \%$ de los encuestados consideran que la competencia esta conseguida, el $21 \%$ que no lo está y el $43 \%$ que necesita mejorar. Su unimos las opciones 2 y 3 , como las más representativas para tener en cuenta que competencias hay que potenciar en nuestro plan formativo, tenemos que el $64 \%$ de los encuestados consideran que hay que mejorar esa competencia. Con respecto a los colectivos podemos observar como son los profesores y los graduados los que mejor creen que esta competencia está de alguna forma conseguida, mientras que los empleadores la mayoría de ellos piensan que o no esta conseguida o que necesita mejorar.

En la competencia o 4 que aparece en la tabla 3 se puede comprobar que el $64 \%$ de los encuestados consideran que la competencia está conseguida totalmente, el $36 \%$ se reparte entre no conseguida o que necesita mejorar. Por colectivo, en este caso son los profesores los que más opinan que deberían mejorar los graduados en esta competencia. Si analizamos los resultados alcanzados en la tabla 3 en la competencia $\mathrm{n}^{\circ} 7$ "Capacidad para utilizar herramientas informáticas", comprobamos que el $36 \%$ de los encuestados consideran que la competencia está conseguida, y que el $64 \%$ se reparten entre no conseguida o que necesita mejorar. Por colectivos podemos observar que son los empleadores los que tienen una postura más crítica sobre esta competencia y que la gran mayoría piensan que los graduados deben mejorar en esta competencia.

El análisis de los resultados de la competencia $n^{\circ} 8$, "Capacidad para escribir y hablar en idiomas extranjeros", que se muestra en la tabla 3 , refleja que el $17 \%$ de los encuestados creen que esta competencia esta conseguida, mientras que el $83 \%$ se reparten entre las opciones de no conseguida o que necesitan mejorar. Por colectivos en este caso son los profesores y los empleadores los más críticos. Los resultados en la competencia 19.- Capacidades técnicas y de orientación a resultados (Concentración y abstracción, orientación de calidad), mostrados en la Tabla 3, fueron que el $47 \%$ de los encuestados consideran que la competencia esta conseguida, mientras que el 53\% reparte sus respuestas entre no conseguida o que necesitan mejorar. Por colectivos los profesores son ligeramente más optimistas con esta competencia. En la competencia no 23, "Habilidades internacionales", una parte de los resultados de la Tabla 3 reflejan que el $25,5 \%$ de los encuestados consideran que la competencia esta conseguida, y el 74,5 que o no esta conseguida o que necesita mejorar. Con respecto a los colectivos en este caso son los profesores y los empleados los más críticos en esta competencia. Este análisis de los resultados refleja que las competencias a desarrollar dentro de nuestro plan formativo fueron:

Tabla 4: Competencias a desarrollar y porcentajes

\begin{tabular}{|l|c|}
\hline \multicolumn{1}{|c|}{ Competencia } & $\begin{array}{c}\% \text { Análisis de } \\
\text { necesidades }\end{array}$ \\
\hline 8.- Capacidad para escribir y hablar en idiomas extranjeros & $83 \%$ \\
\hline 23.- Habilidades internacionales & $74,5 \%$ \\
\hline 1.- Capacidades técnicas y específicas, Dominio de su área o disciplina & $64 \%$ \\
\hline 7.- Capacidad para utilizar herramientas informáticas & $64 \%$ \\
\hline $\begin{array}{l}\text { 19.- Capacidades técnicas y de orientación a resultados, Concentración y abstracción, orientación de } \\
\text { calidad }\end{array}$ & $53 \%$ \\
\hline 4.- Pensamiento analítico & $36 \%$ \\
\hline
\end{tabular}

Con respecto a las competencias específicas derivadas de las anteriormente estudiadas se analizaron las relacionadas con la competencia ํㅜㄷ y la competencia ํㅜ 7 como las más concretas a desarrollar en nuestro plan formativo, ya que resto se desarrollaron de forma transversal en todos los cursos de nuestro plan formativo. Las competencias específicas fueron las mostradas en la Tabla 5. 
Tabla 5: Relación de competencias específicas a desarrollar

Adquisición de conocimientos de regulación automática y técnicas de control y su aplicación a la automatización industrial

Capacidad para diseñar sistemas de control y automatización industrial.

Capacidad para conocer e identificar las variables de un sistema controlado y los objetivos de control

Adquisición del conocimiento del funcionamiento de los PID, sus ámbitos de aplicación y problemática asociada.

Capacidad para trabajar con varias estructuras de control diferentes y conocer sus distintas aplicaciones.

En resultado del análisis de necesidades formativas se recogieron 8 cursos que se repartieron en dos semestres con el objetivo de mejorar el currículo y así la formación de los estudiantes del Grado de Ingeniería en la rama de Ingeniería de Sistemas y Automática (ISA). En el primer semestre los cursos fueron: ISE1_Introducción a sistemas industriales; ISE2_Sistemas automatizados; ISE3_Electrónica para sistemas industriales; ISE4_Comunicaciónes industriales y en tiempo real. En el segundo semestre los cursos fueron: ISE5_Diseño de controladores industriales de alto nivel; ISE6_Controladores industriales inteligentes; ISE7_Sistemas de control y proco adaptativo. Diseño, métodos y estrategias de control; ISE8_Sistemas de control y proceso adaptativo. Reguladores y comunicación. El estudio piloto a través del cual se midieron los indicadores indicados, se realizó con dos cursos en concreto con el ISE7 e ISE8, los contenidos de los cursos fueron los siguientes:

ISE7: Sistemas de Control y Proceso Adaptativo. Diseño y métodos y estrategias de control: Función de transferencia de un sistema. Sensibilidad y precisión. Función de transferencia de un Sistema. Diagramas de bloques y diagramas de flujo. Sensibilidad, precisión y fiabilidad. Perturbaciones y errores. Controlabilidad y observabilidad .Controlabilidad y observabilidad. Métodos de selección de variables de estado. Fiabilidad y estabilidad. Criterios de estabilidad.Sistemas en cadena abierta y en cadena cerrada.

ISE8: Sistemas de Control y Proceso Adaptativo. Reguladores y Comunicación. Controladores y calibración. Testeado funcional. Sistemas de Control y Proceso Adaptativo. Reguladores y Comunicación. PID. Condiciones de operación .Controladores PID. Control Adaptativo Predictivo Experto.

Los resultados del estudio piloto en relación con los distintos indicadores son mostrados en las Tablas 6 y 7 y en las Figura 4 y 5 . Se comparan todos los indicadores que hemos medido en los cursos ISE7 e ISE8 para comprobar cuál de ellos ha sido el más valorado por los estudiantes.

Tabla 6: Comparación de indicadores del estudio piloto

\begin{tabular}{|c|c|c|c|c|c|}
\hline & Expectativas/motivación & Utilidad & $\begin{array}{c}\text { Contenido/ } \\
\text { Metodología }\end{array}$ & Herramientas & \\
\hline $\begin{array}{c}\text { Media } \\
\bar{X}\end{array}$ & 4,29 & 4,2 & 4,29 & 4,35 & $\bar{X}=4,28$ \\
\hline
\end{tabular}

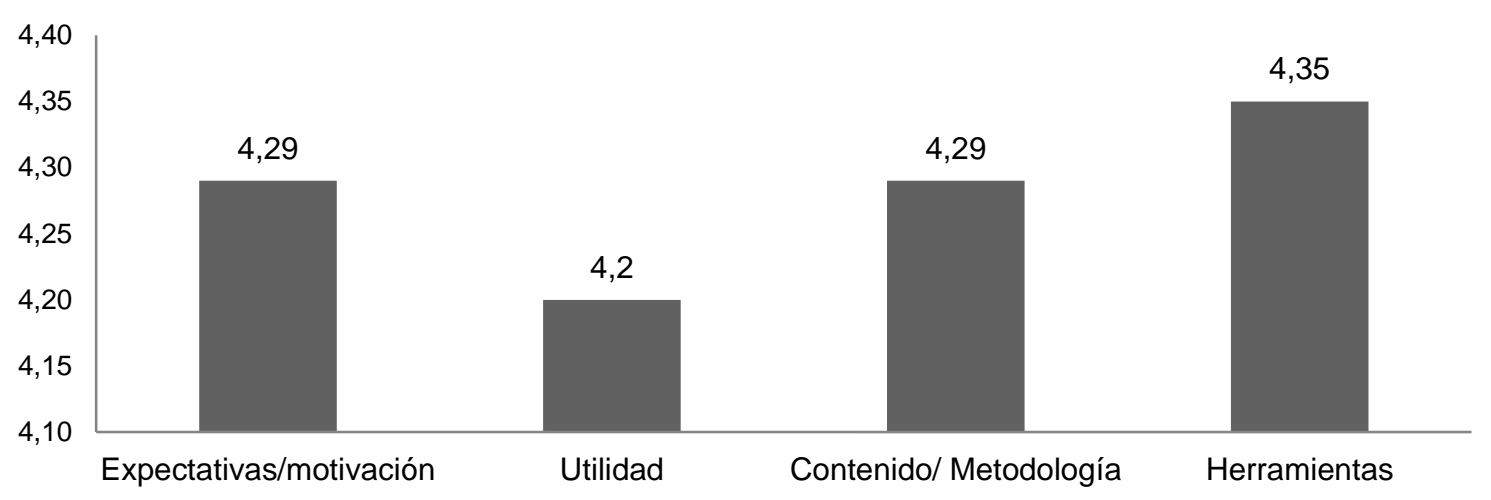

Fig. 1: Comparación de medias entre indicadores

A la vista de los resultados se puede comprobar como todos los indicadores obtienen puntuaciones superiores a 4 sobre un total de 5 . Si analizamos los datos con detalle observamos como el indicador mejor valorado por los estudiantes ha sido el de herramientas con un 4,35; seguido por el de contenidos/metodología y Expectativas/motivación con un 4,29 respectivamente, y muy cercano se encuentra el indicador de utilidad con un 4,2 . 
Tabla 7: Comparación de indicadores y fases de los indicadores en los cursos ISE7 e ISE8

\begin{tabular}{|l|c|c|c|}
\hline & Fase pre $\bar{X}$ & Fase post $\bar{X}$ & Cursos ISE7 e ISE8 $\bar{X}$ \\
\hline Expectativas/Motivación & 3,75 & 3,35 & 4,29 \\
\hline Utilidad & 3,84 & 3,65 & 4,20 \\
\hline Contenidos/Metodología & 4,24 & 3,6 & 4,29 \\
\hline Herramientas & 4,5 & 3,4 & 4,35 \\
\hline
\end{tabular}

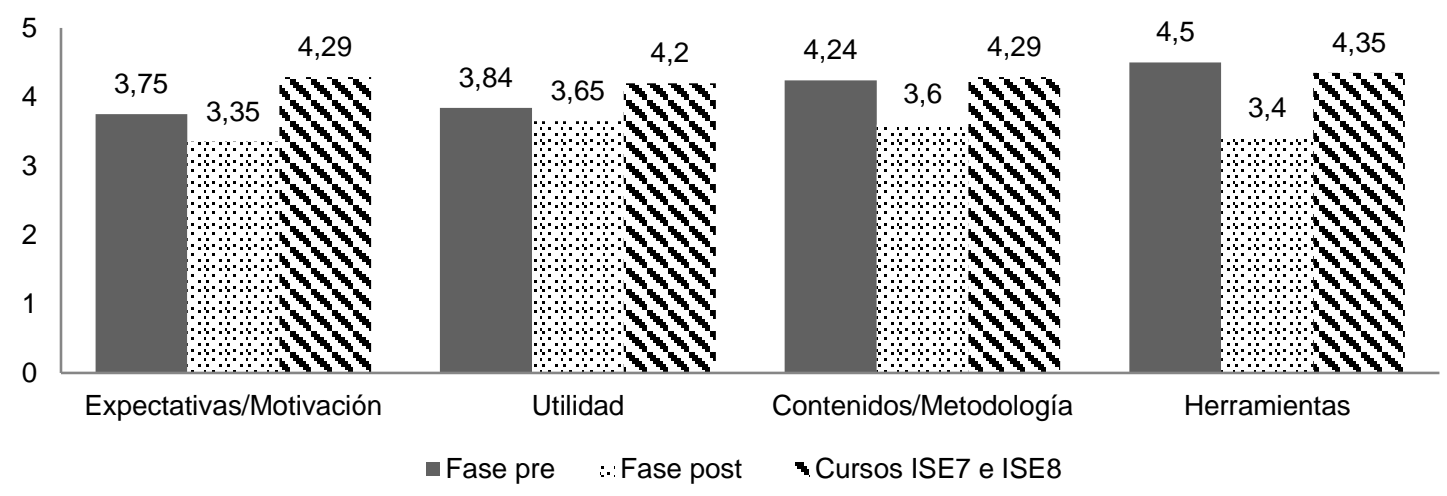

Fig. 2: Comparación entre las fases pre-post y cursos ISE7 e ISE8

Si analizamos los datos podemos comprobar que las puntuaciones medias de los indicadores suben en esta fase final del proyecto con la realización de los cursos. Los estudiantes, después de realizar los cursos ISE7 e ISE8, aumentan las puntuaciones medias de todos los indicadores. De forma detallada, en el análisis por indicadores, podemos comprobar como el indicador expectativas/motivación en una primera fase del proyecto obtiene una puntuación de 3,75, en la segunda fase o fase post baja a un poco y obtiene una puntuación de 3,35, pero cuando los alumnos realizan los cursos ISE7 e ISE8 sus puntuaciones suben hasta un 4,29 sobre 5 . Con respecto al indicador utilidad, su comportamiento es el mismo, en una fase inicial los estudiantes conceden a este indicador una puntuación de 3,84, en una segunda fase una puntuación media de 3,65 subiendo hasta 4,20 después de la realización concreta de los cursos ISE7 e ISE8. Con respecto al indicador metodología observamos que en la fase inicial la puntuación fue de 4,24; la puntuación baja en la fase post con un 3,6 subiendo posteriormente después de la realización de nuestros cursos hasta un 4,29. En el indicador herramientas observamos como en una fase inicial la valoración de los estudiantes es de 4,5 sobre 5; en la fase post la puntuación baja a 3,4 y cuando los alumnos realizan los cursos ISE7 e ISE8 la puntuación vuelve a subir hasta un 4,35 sobre 5. De acuerdo a los resultados se puede afirmar que las valoraciones de los estudiantes, después de realizar los cursos ISE7 e ISE8 aumentas sus puntuaciones y, por tanto, su valoración sobre el sistema PAC.

\section{DISCUSIÓN}

El presente proyecto de investigación ha presentado unas bases sólidas para la formulación de un diseño de plan de formación de Master proporcionando la información relevante en torno a las competencias clave que deben ser desarrolladas en el mismo, y a su vez teniendo presente a todos los actores implicados para la consecución de la empleabilidad en la rama de ingeniería de Sistemas y Automática, a través de una perspectiva integrada de la académica y la industria. Las competencias mejor valoradas han sido ํo1.Capacidades técnicas y específicas, Dominio de su área o disciplina. Profesores y empleadores coinciden además en la competencia no 4.- Pensamiento analítico. Por su parte empleadores y graduados coinciden en la competencia 7.- Capacidad para utilizar herramientas informáticas; en la ํㅡ 8.- Capacidad para escribir y hablar en idiomas extranjeros, en la ㄲo 19.- Capacidades técnicas y de orientación a resultados, concentración y abstracción, orientación de calidad; y en la no 23 Habilidades internacionales.

De la misma forma el presente trabajo presenta otras competencias a desarrollar al igual que otras investigaciones como las realizadas por Ashman et al. (2008) y Nair, Patil, y Mertova (2009), la competencia del trabajo en equipo es considerada importante dentro del campo de la ingeniería. Por otra parte, nuestra investigación, al igual que Rué (2009), asegura que es necesario que se incluyan una serie de competencias profesionales dentro del currículum académico, que deben centrarse en torno al: aprendizaje autónomo, la creatividad, el pensamiento crítico y resolución de problemas, habilidades de comunicación social, trabajo en grupo; por esta razón, el diseño del master se centra en el desarrollo de las habilidades de aprendizaje que les permitan continuar estudiando de un modo que habrá de ser en gran medida autodirigido o autónomo por parte del estudiante. 
Otras investigaciones, como la llevada a cabo por Peng, Shulin y Gu (2014), encuentra también diferencias entre el grado de satisfacción del desarrollo de competencias en el mundo laboral, frente al grado de satisfacción que tienen los estudiantes de grado, empleadores y profesores. En este caso es especialmente relevante cómo los empleadores y graduados están más en línea en las competencias que deben mejorar o no están suficientemente desarrolladas puede atribuirse al hecho de que estas competencias clave son muy tenidas en cuenta en las empresas, mientras que en el mundo universitario son competencias que se han desarrollado de forma transversal.

Una de las competencias que requiere toda la atención por los resultados obtenidos es la Capacidad para utilizar herramientas informáticas. Aunque los recién graduados consideran que esta competencia es clave dentro del mundo laboral actual, en los resultados muestran que necesitan mejorar Este resultado no choca en contraposición con el cuestionario realizado por los empleadores, donde también aseveran que dicha competencia es una de las más importantes, y consideran que la preparación que tienen los recién graduados en esta competencia necesita mejorar Este mismo hecho ya ha sido remarcado por otras investigaciones, como las elaboradas por Peng, Shulin y Gu (2014) y Rugarcía et al. (2000).

Ante los datos obtenidos en otras investigaciones y las conclusiones obtenidas en los resultados de esta investigación, se han formulado una serie de competencias generales y específicas que son incluidas dentro del diseño y sistematización del master como elementos fundamentales que se deben tener con especial consideración: Se consideraron como las mejor valoradas: Capacidades técnicas y específicas, dominio de su área o disciplina; Pensamiento analítico; Capacidad para utilizar herramientas informáticas; Capacidad para escribir y hablar en idiomas extranjeros; Capacidades técnicas y de orientación a resultados, concentración y abstracción, orientación de calidad y Habilidades internacionales. De todas estas el análisis reflejo que: En la competencia: Capacidades técnicas y específicas (Dominio de su área o disciplina). $64 \%$ de los encuestados consideran que no está conseguida o que necesita mejorar Con respecto a los colectivos podemos observar como son los profesores y los graduados los que mejor creen que esta competencia esta de alguna forma conseguida, mientras que los empleadores la mayoría de ellos piensan que o no esta conseguida o que necesita mejorar.

En la de pensamiento analítico.- $36 \%$ se reparte entre no conseguida o que necesita mejorar. Por colectivo en este caso son los profesores los que más opinan que deberían mejorar los graduados en esta competencia. En la de Capacidad para utilizar herramientas informáticas.- el $64 \%$ se reparten entre no conseguida o que necesita mejorar. Por colectivos son los empleadores los que tienen una postura más crítica sobre esta competencia y que la gran mayoría piensan que los graduados deben mejorar en esta competencia. En la de Capacidad para escribir y hablar en idiomas extranjeros el $83 \%$ se reparten entre las opciones de no conseguida o que necesitan mejorar. Por colectivos, en este caso, son los profesores y los empleadores los más críticos. En la de Capacidades técnicas y de orientación a resultados, concentración y abstracción, orientación de calidad, los resultados en esta competencia fueron que el $53 \%$ reparte sus respuestas entre no conseguida o que necesitan mejorar. Por colectivos, los profesores son ligeramente más optimistas con esta competencia. En la de Habilidades internacionales En esta competencia los resultados reflejan que el 74,5 que o no esta conseguida o que necesita mejorar. Con respecto a los colectivos, en este caso, son los profesores y los empleados los más críticos en esta competencia.

Como competencias específicas: Adquisición de conocimientos de regulación automática y técnica de control y su aplicación a la automatización industrial; Capacidad para diseñar sistemas de control y automatización industrial; Capacidad para conocer e identificar las variables de un sistema controlado y los objetivos de control; Adquisición del conocimiento del funcionamiento de los PID, sus ámbitos de aplicación y problemática asociada; Capacidad para trabajar con varias estructuras de control diferentes y conocer sus distintas aplicaciones. Las relacionadas con el idioma extranjero y las relaciones internacionales, se desarrollar a través de la propia metodología ofreciendo el Master en distintos idiomas y de forma internacional, los alumnos podían estar conectados en remoto a las distintas actividades y desde diferente partes de Europa.

Al igual que otros estudios del área, como el realizado por Fiad y Galarza (2015), la estrategia de aprendizaje está orientada a que los estudiantes puedan analizar y participar de forma activa en las tareas de aprendizaje utilizando para ello el uso de casos prácticos y mapas mentales que son empleados en otros ámbitos, como en el diseño de la ingeniería de software. El modelo de flujo de trabajo PAC está en línea con el enfoque de resolución de problemas, y además, el modelo de flujo de trabajo PAC refleja una investigación basada en modelos de desarrollo de aprendizaje cooperativo. Y de esta forma se atiende a las competencias clave que se habían detectado que debían ser atendidas con mayor urgencia y que en cuestión fueron La capacidad de trabajo en equipo; Capacidad para utilizar herramientas informáticas; Capacidad de gestión del tiempo; Habilidades de organización; Capacidad de crítica y autocrítica. 
De entre estas competencias antes nombradas, destacó de forma relevante atender de forma específica a la capacidad para utilizar las herramientas informáticas las siguientes cuestiones: Comprensión de las reglas básicas de la programación; Nociones sobre las reglas básicas para crear la interfaz de usuario; Conocimiento de los métodos de diseño del programa en la empresa aprobados; Conocimientos sobre el programa de software específico. Por su parte la valoración de los distintos indicadores tanto en la fase pre como en la fase post de la fase piloto de los cursos sometidos a estudio, ISE7 e ISE8, fue muy positiva en todos sus parámetros, Motivación/expectativas; Utilidad; Contenidos/metodología; Herramientas, tanto en la fase pre como en la post.

\section{CONCLUSIONES}

El Plan de Formación de PAC se compone de una secuencia de actividades para apoyar a los formadores en el diseño de los planes de estudios propio del Master. El formato del modelo se basa en investigaciones recientes sobre el diseño y el desarrollo del aprendizaje de apoyo al rendimiento. A partir de esta fase del proyecto de investigación PAC, se pone de manifiesto la necesidad de desarrollar una serie de competencias clave a través de la formación de postgrado de los graduados en ingeniería. $Y$ bajo esta premisa el proyecto PAC considero estos resultados para el diseño y puesta en marcha de un plan de formación a nivel de maestría para detectar una posible solución para hacer frente a las diferencias de competencias entre los requerimientos del mundo laboral y las empresas, frente a los resultados obtenidos por los recién graduados. Sería motivo de futuras investigación analizar la evolución del impacto social, y a nivel de empleabilidad, que ha tenido el desarrollo de las competencias analizadas en los graduados en el master diseñado a la hora de conseguir su empleo y en la mejora de su puesto de trabajo.

\section{REFERENCIAS}

ABET, Criteria for Accrediting Engineering Programs: Effective for Evaluations During the 2010-2011 Accreditation Cycle, ABET, Baltimore, EEUU (2009)

Agudo, J.E. y otros tres autores, Competencias transversales: Percepción de su desarrollo en el grado de ingeniería en diseño industrial y diseño de productos, doi http://dx.doi.org/10.4067/S071850062013000500006, Revista Formación Universitaria (en línea), 6 (5), 39-50 (2013)

Ashman, P. J. y otros cuatro autores, Stakeholder Perceptions of Chemical Engineering Graduate Attributes at the University of Adelaide. Proceedings of the Chemeca 2008 Conference: Towards a Sustainable Australasia (912-921), Australia, 28 September-1 October (2008)

Barrios, T., J. Lencina, y M. Marín, Integración de Laboratorios Virtuales en Moodle, Actas del Congreso Iberoamericano de Ciencia, Tecnología, Innovación y Educación, Buenos Aires, Argentina, Noviembre (2014)

Blancero, D., J. Boroski, y L. Dyer, Key competencies for a transformed human resource organization: Results of a field study, doi: 10.1002/(SICI)1099-050X(199623)35:3<383::AID-HRM6>3.0.CO;2-T, Human Resource Management (en línea), 35 (3), 383-403 (1996)

Bodmer, C. y otros tres autores, SPINE: Successful Practices in International Engineering Education International Benchmarking Study. Final Report. Project initiated by the Board of the Swiss Federal Institutes of Technology and Engineers shape our future, https://goo.gl/jLdv8c, Swiss Federal Institutes of Technology and Engineers (2002)

Bustamante M.L. y otros tres autores, Fundamentos de la enseñanza por competencias a nivel de postgrado en dos universidades públicas Chilenas, doi http://dx.doi.org/10.4067/S0718-50062015000600004, Revista Formación Universitaria (en línea), 8 (6), 23-30 (2015)

Caldas, M. E., G. Lacalle, y R. Carrión, Políticas de formación de recursos humanos (Recursos humanos y responsabilidad social corporativa), Editex, ebook (2012)

Carozo Martín, V., La formación profesional para el empleo y su influencia en la edad de la población activa de Andalucía, en J. Domingo, E. M. Olmedo, y D. Amber (coords.), Investigación en Ciencias de la Educación: I Jornadas Doctorales en Cs. de la Educación, http://hdl.handle.net/10481/30580, U. de Granada, Granada (2013)

Downey, G. L., J.C. Lucena, \& C. Mitcham, Engineering Ethics and Identity: Emerging Initiatives in Comparative Perspective, doi: 10.1007/s11948-007-9040-7, Science and Engineering Ethics (en línea), 13 (4), 463-487 (2007) 
EAAB, Engineers Australia Policy on Accreditation of Professional Engineering Programs, EAAB, Canberra, Australia (2005)

ECUK, The UK Standard for Professional Engineering Competence (UK-SPEC) Chartered Engineer and Incorporated Engineer Standard, ECUK, Londres, Reino Unido (2003)

Estepa, R. M., y A. J. Estepa, Trabajar con la incertidumbre del mundo laboral: análisis de una experiencia con Aprendizaje Basado en Problemas en Redes de Ordenadores, http://redaberta.usc.es/redu, ISSN 18874592, REDU- Revista de Docencia Universitaria, 9 (2), 213-232 (2011)

Farrell, D. y otros cuatro autores, The Emerging Global Labor Market: Part II - the Supply of Offshore Talent. McKinsey Global Institute, San Francisco, EEUU (2005)

Heywood, J., Engineering Education. Research and Development in Curriculum and Instruction, IEEE Press, ISBN: 978-0-471-74111-4, John Wiley, EEUU (2005)

IES, Engineering Accreditation Board: Accreditation Manual. IES, Singapore (2004)

Jiménez, M. y otros tres autores, Dilemas Ideológicos ante una Formación Universitaria con Sello Valórico Distintivo. El Caso de una Carrera de Ingeniería, doi: 10.4067/S0718-50062013000400003, Revista Formación Universitaria (en línea), 6(4), 13-26 (2013)

Lucena, J., y otros tres autores, Competencies Beyond Countries: The Re-organization of Engineering Education in the United States, Europe, and Latin America, doi: 10.1002/j.2168-9830.2008.tb00991.x, Journal of Engineering Education (en línea), 97 (4), 433-47 (2008)

Marchisio, S., F. Lerro y O. Von Pamel, Empleo de un laboratorio remoto para promover aprendizajes significativos en la enseñanza de los dispositivos electrónicos, http://www.sav.us.es/pixelbit/actual/10.html, ISSN 1133-8482, Pixel-Bit. Revista de Medios y Educación, 38 (1) enero, 129-139 (2011)

Fiad, S B. y O. D. Galarza, El Laboratorio Virtual como Estrategia para el Proceso de EnseñanzaAprendizaje del Concepto de Mol, doi: http://dx.doi.org/10.4067/S0718-50062015000400002, Revista Formación Universitaria (en línea), (4), 03-14 (2015)

Martin del Peso, M., A.B. Rabadán Gómez, y J. Hernández March, Desajustes entre formación y empleo en el ámbito de las Enseñanzas Técnicas universitarias: la visión de los empleadores de la Comunidad de Madrid, doi: 10.4438/1988-592X-RE-2011-360-110, Revista de Educación (en línea), № 360, 244-267 (2013)

Nair, C. S., A. Patil, y P. Mertova, Re-engineering Graduate Skills - a Case Study, doi: 10.1080/03043790902829281, European Journal of Engineering Education (en línea), 34 (2), 131-39 (2009)

NBA, AICTE, Accreditation Parameter: Criteria and Weightages. National Board of Accreditation, Delhi, India (2000)

Peng, L., Z. Shulin, y J. Gu, Evaluating the competency mismatch between Master of Engineering graduates and industry needs in China, doi: 10.1080/03075079.2014.942268, Studies in Higher Education (en línea), 41 (3), 445-461 (2014)

Pimienta, J. H., Secuencias didácticas: aprendizaje y evaluación de competencias en educación superior, http://dialnet.unirioja.es/servlet/articulo?codigo=3601028, ISSN: 0210-5934, Bordón, Revista de Pedagogía, 63 (1), 77-92 (2011)

Rué, J., El aprendizaje Autónomo en Educación Superior, Narcea, Madrid, España (2009)

Rugarcia, A. y otros tres autores, The Future of Engineering Education. I. A vision for a new century, http://www4.ncsu.edu/unity/lockers/users/f/felder/public/Papers/Quartet1.pdf, ISSN: 00092479, Chemical Engineering Education, 34 (1), 16-25 (2000)

Schmal, R., Evolución de un programa de formación en competencias genéricas, doi: 10.4067/S071850062015000600012, Revista Formación Universitaria (en línea), 8(6), 95-106 (2015)

Sgobbi, F. \& F. Suleman, A Methodological Contribution to Measuring Skill (Mis) Match, doi: 10.1111/j.14679957.2012.02294.x, The Manchester School (en línea), 81 (3), 420-37 (2013)

Tuning, Tuning Educational Structure in Europe. Informe final de la segunda fase del proyecto, https://goo.gl/BPgkL2, Tunning (2005) 
